\title{
Clinical Approaches to the Out-of-Body Experience
}

\author{
Stuart W. Twemlow, M.D. \\ Topeka Institute for Psychoanalysis \\ University of Kansas
}

ABSTRACT: The author reviews aspects of the out-of-body experience (OBE) related to psychic experiences and personality traits, and describes a continuum of experiences of altered mind/body perception, from the prototypical $\mathrm{OBE}$ on the healthy end to schizophrenia and organic brain syndromes on the other end. The impact of the OBE on the individual's life is described, with suggestions for a psychoeducational approach to the clinical management of the patient with an OBE to allow maximum growth from the consciousnessexpanding effects of the experience.

The out-of-body experience (OBE) has had a remarkably ambivalent relationship with parapsychology. Strictly speaking, the $\mathrm{OBE}$, in its most common form, shows none of the esoterica usually subsumed under the rubric extrasensory perception or psychic phenomena but merely describes an unusual perception of the relationship between the subject's mind and his or her body. In the widely publicized researches of famous spiritualists and psychics such as Sylvan Muldoon and Hereward Carrington (1969), Robert Monroe (1977), and many others, out-of-body "travel" is a term than has been used to describe very esoteric planes of reality. For theosophists, it is a psychic state fundamental to the mapping and charting of the astral plane and other

Dr. Twemlow is in the private practice of psychiatry in Topeka, and is on the faculty of the Topeka Institute for Psychoanalysis, the Menninger Foundation, and is Clinical Associate Professor of Psychiatry at the University of Kansas School of Medicine in Kansas City and Wichita. Requests for reprints should be addressed to Dr. Twemlow at 5040 S.W. 28th Street, Topeka, KS 66614. 
realities. In this paper, I will consider the management of the experience as it presents to clinicians for treatment. As will be seen from the previous research of Glen Gabbard and myself (Gabbard \& Twemlow, 1984), we do not consider the OBE to be a disease state, but a normal experience usually requiring no specific clinical intervention beyond reassurance and education. The typical OBE is a pleasurable experience unlikely to present specifically to a clinician for treatment. However, experience over the years with frequent consultations with psychiatrists, psychologists, and other clinicians indicates that sometimes OBEs appearing in the context of psychoanalysis or psychotherapy, or appearing spontaneously in patients undergoing treatment for other conditions, arouse the clinician's curiosity and strong urge to intervene. Unfortunately, the more medically oriented clinicians tend to wish to "treat" the out-of-body state, rather than allowing the individual to experience and profit from it. Because of this, there is value in considering differentiation of the OBE from pathological states.

Even clinicians sympathetic to mystical and esoteric states can pathologize the OBE. Raymond Kennedy (1976) called the OBE a "selfinduced depersonalization experience"; although in his case report he indicates that he was personally studying Hindu mystical disciplines, he used a pathologizing depersonalization model to describe the OBE. Harvey Irwi. (1985), in his book Flight of Mind, gave several other instances of the classification of an OBE as examples of autoscopy or depersonalization. D.H. Rawcliffe (1959) connected the experience with psychosis and hysteria, although our studies (Gabbard \& Twemlow, 1984) contradicted that finding. Herbert Greenhouse (1975) related a case in which a psychiatrist agressively ordered an out-of-body experiencer into involuntary psychiatric examination on the basis of that experience alone!

\section{What Is an Out-of-Body Experience?}

In our previously reported questionnaire research of 339 out-of-body subjects (Twemlow, Gabbard, and Jones, 1982), we found the following profile description of an OBE: the experience occurs in a state of physical relaxation and mental calmness without emotional stress. The experience is pleasant and the subject typically feels calm, peaceful, and quiet. Occasionally, ecstatic experiences of joy may occur. Very unpleasant feelings are much less common, with the feeling of going crazy being rare. The subject is likely to find himself or herself in the same environment as the physical body, which he or she sees in an 
inert state below the point of perception, which is spatially separate from the physical body. The subject usually notes that he or she has a "new body" in a form similar to the physical body. The experience is vivid in quality, is more real than a dream, and has a profound influence on the individual's subsequent life according to self-report. Frequently, the subject may view the experience as spiritual in nature, and may be more likely to believe in life after death as a result of the experience. The individual has a fascination with the experience and would like to try it again, often describing it as one of the greatest events of his or her life. A prototypical OBE in composite form, of course, only describes a majority of experiences and not those extensive numbers of variations from this prototype.

From a more theoretical and philosophical point of view then, our research enabled us to define the $\mathrm{OBE}$ as "an altered state of consciousness in which the subject feels that his mind or self-awareness is separated from his physical body and that sense of self-awareness is more vivid and more real than a dream" (Gabbard \& Twemlow, 1984). This definition is quite similar to others reported in the field including those of Irwin (1985) and John Palmer (1978).

\section{OBE and Other Psychic Experiences}

Palmer (1978) stated that there is an association between the OBE and various psychic phenomena. He particularly referred to apparitional phenomena. In our study of 339 subjects, we found that $76 \%$ of the subjects felt they had experienced mental telepathy; $21 \%$ felt they had been healed by a psychic; 30\% felt they had healed others by psychic means; $32 \%$ had seen a UFO; $67 \%$ had a precognitive dream; $59 \%$ felt they had communicated with an animal; and $51 \%$ felt they had had contact with a person after he or she had died. Additionally, $67 \%$ of the population claimed they had had blood relatives who experienced ESP and 29\% had had blood relatives who had an OBE. These findings indicate an association of $\mathrm{OBE}$ and other psychic experiences, although the incidence of psychic experiences in the U.S. population is as yet undetermined but is likely to be significant. In a random sample of the population of Iceland, Erlendur Haraldsson, Asa Gudmundsdottir, Asthor Ragnarsson, Johann Loftsson, and Sigtryggur Jonsson (1977) found about 66\% of the sample had had some form of ESP experience. That study is unique with regard to the representativeness of the sample. 


\section{OBE and Personality}

According to our literature review, between 10 and $25 \%$ of the population have experienced an OBE (Gabbard \& Twemlow, 1984).

The clinical management of the OBE may well be improved by a knowledge of personality and psychopathological traits of OBE, if any were to exist. However, most of the studies of OBEs are not contributory in this regard. Cognitive dimensions, for example, attention/ perceptual dimensions, such as absorption (Irwin, 1985), may distinguish out-of-body experiencers. In our study of OBE subjects, we did not find that measures of absorption, hysteroid tendencies, psychoticism, or death anxiety enabled us to distinguish the subjects from our control subjects who had not had the experience but who had a high interest in the OBE (Twemlow, Gabbard, and Jones, 1982). One exception was that OBE subjects were significantly less danger-seeking than the comparison group, although Irwin's study (1985, p. 198) did not support that finding. In a subsequent study, out-of-body experiencers were compared to 678 subjects used in a study of emotional wellbeing of nonpatient populations (Gabbard \& Twemlow, 1984); 121 of those subjects were studying transcendental meditation, 96 were college students, 366 were people enrolled in an altered state of consciousness training program that has as one of its goals induction of an OBE, and 42 were participants in a conference on altered states of consciousness, most of whom were mental health professionals. Our OBE subjects had significantly greater emotional health than the patient group and the transcendental meditation trainees, and better emotional adjustment than the control group of randomly selected college students. In fact, their psychological health was comparable to the workshop attendees and mental health professionals. Thus, we concluded from this and other demographic and psychological data that the typical OBE subject is an average, healthy American, and may be of any age, sex, or marital status, with a variable religious background, but with a history of changing from traditional religions to rather nontraditional religious pursuits after the experience. In general, most studies suggest that the out-of-body experiencer is not any more neurotic than the nonexperiencer, and data from personality inventories does not delineate a clear profile of the subject. A recent study by Jerome Tobacyk and Thomas Mitchell (1987) showed that reporters and nonreporters of OBEs were virtually indistinguishable on the criteria they used. They studied 445 college students, including 65 who had had OBEs. Extensive testing on the subjects included studies of death orientation, defensive style, narcissism, self-concept, and social desirability. They concluded, as most studies do, that the OBE is "a normative, non- 
psychopathological human experience" (Tobacyk \& Mitchell, 1987, p. 369).

Before I discuss the differentiation of the OBE from psychopathological states, I would like to emphasize, as we have previously (Gabbard \& Twemlow, 1986, p. 354), "while the OBE respondents in our study were characterized by psychological health, that fact in no way excludes the possibility that psychiatrically disturbed individuals can also report OBEs. An OBE is a nonsymptomatic altered state of consciousness that is not necessarily confined to normal subjects. In contrast to an OBE, schizophrenia is a constellation of symptoms that form a psychiatric syndrome. The important point is that an OBE is not prima facie evidence of significant psychiatric illness."

\section{States of Altered Mind/Body Perception}

The clinical approach to the management of OBE is best considered by using the concept of altered mind/body perception. This phrase (Gabbard \& Twemlow, 1986) refers to a group of phenomena that all have in common an altered state of consciousness in which there is a subjectively perceived distortion of the normal spatial relationship between the mind and the body. Clinically, this assortment of "out-ofbody" states must be differentiated before problem management can be instituted. The altered mind/body perception concept allows these phenomena to be considered on a continuum with the prototypical, nonpathological OBE on the healthy end and the disintegrated highly ego dystonic state of schizophrenia on the other end of the continuum. In between, there are an assortment of out-of-body states that are variants on normality or indicators of frank psychopathology.

\section{OBE and Psychopathological States}

\section{Depersonalization}

Depersonalization disorder is a dissociative phenomenon, defined as an alteration in the perception of the self so that the feeling of one's own reality is temporarily lost. This feeling of unreality may refer to an estrangement from the self, from the body, or from one's own surroundings. Derealization refers specifically to the estrangement from one's environment, although derealization is ordinarily part of the depersonalization disorder. Depersonalization may take many 
forms: one may feel that one's body is dead or numb; one may feel that a certain body part or parts, such as the hands or feet, are not connected with the rest of one's body; one may feel detached from one's self image so that one does not know who one is; or, one may have the sense that one is observing oneself at some distance. The latter variant of depersonalization is most commonly confused with OBE. It should be obvious then that the depersonalizer does not necessarily feel out of the body. Russell Noyes, Paul Hoenk, Samuel Kuperman, and Donald Slymen (1977) found that feelings of frank detachment from the body are quite infrequent, characterizing only $19 \%$ of psychiatric patients who suffer from depersonalization. So in depersonalization, there is in fact a split between the observing and functioning self even if one does not feel truly separated from one's body. A victim of an automobile accident might report that he or she experienced the entire accident as though it were happening to someone else, or that he or she saw the functioning self go through the motions of turning the wheel and stomping on the brakes. Studies of depersonalization have consistently linked it to dream-like states and have noted that it occurs as a symptom in a number of illnesses, including depression, schizophrenia, and anxiety (Fisher \& Seidner, 1963).

The clinician can differentiate depersonalization from OBEs based on several key features. Depersonalizers do not feel physically detached, but instead feel unreal or numb. The out-of-body experiencer does not experience a split between the observing and functioning selves, but typically feels integrated in a point of perception outside the physical body, which is usually viewed as non-functional. The depersonalization state is extremely unpleasant, whereas the OBE is usually a calm and pleasant, even a joyous state not accompanied by feelings of disintegration. The depersonalizer is usually in a dreamlike state; whereas the opposite is true of the out-of-body subject, who is quite definite that the experience is not a dream. Out-of-body experiencers are frequently in a relaxed state and depersonalizers hyperaroused. Although other features may be present, these main features should enable the clinician to distinguish these two states with the treatment obviously being focused on the underlying condition.

\section{Autoscopy}

Technically, autoscopy is not a true form of altered mind/body perception (Gabbard \& Twemlow, 1986) because the mind remains identified with the body; however, the literature often confuses autoscopic 
phenomena with OBEs because the OBE subject might well believe that he or she is seeing his or her own body from an altered perceptual vantage point. A key differential point is that the person in the midst of an OBE feels that the mind and perceptual point have shifted to a location spatially distinct form the physical body, which is looked upon as real rather than imaginary. In contrast, the mind and body in autoscopy remain identified with one another, and the subject does not think he or she is perceiving the actual body below. Rather, the double is experienced as an illusory phantom of him- or herself. Autoscopic doubles are also transparent and colorless in contrast to the OBE subject, and only the face and torso are usually seen in autoscopy, often mimicking the subject's movements, particularly the facial expressions. The out-of-body subject often is likely to see an entire body, usually motionless. Positive states of mind are associated with the OBE, while sadness is most commonly associated with autoscopy. Autoscopic phenomena are almost always associated with underlying illness, such as pyrexia, or brain pathology, in contrast with the healthy OBE.

\section{Schizophrenic Body Boundary Disturbances}

Although 5\% of our original reference sample felt that the out-ofbody experience is one of insanity or going crazy, when examined more closely, feeling crazy and being schizophrenic or severely psychotic are two very different states. In a recent survey of OBEs in schizophrenic subjects, Susan Blackmore (1986) interviewed 71 schizophrenic subjects in comparison with 40 control subjects who were randomly selected from a hospital accident ward. The incidence of OBEs in schizophrenics was $14 \%$, which is rather similar to the control group and the general population at large (Gabbard \& Twemlow, 1984). The other symptoms of schizophrenia, including perceptual distortions, were no more common in the OBE schizophrenic group than the non-OBE schizophrenic group, which is in line with our findings that body image distortions are not in any way related to the typical OBE.

We have distinguished (Gabbard \& Twemlow, 1984) seven general categories of body boundary disturbance in schizophrenic individuals: (1) fusion phenomena; (2) the experience of one's body boundaries as constantly changing; (3) underestimation of one's own body size; (4) omission of hands or feet as part of the body image; (5) overestimation and underestimation of the size of body parts; (6) the feeling that one's body is not one's own; and (7) the feeling that one's body is unreal or a 
machine. None of these disturbances includes an alteration of the spatial relationship between the mind and the body. The schizophrenic individual's subjective reports may nevertheless be confused with the OBE of psychologically healthy persons; thus it is necessary to distinguish these disturbances of normal body boundaries clearly from those that stem from developmental failure to establish these boundaries as in schizophrenia. In addition, some clinicians may dismiss the OBE as merely delusional or hallucinatory and prescribe medications and other treatments that are potentially dangerous to the individual, as well as not helpful in ameliorating the symptom. The sort of confusion an uninformed clinician may make between the out-of-body and schizophrenic identity disturbance is well illustrated by a case reported in our study, from which I quote: "Miss G. was a 22-year-old severely schizophrenic woman who was seen ... in intensive psychotherapy for a 4 year period. She had experienced a number of 'fusion' phenomena during that time, manifested by repeatedly misidentifying in others attributes of herself and attributes of those around her in herself. During one psychotic episode of this type, she misidentifies her experience as an out-of-body experience. In the patient's own words, 'It was like an out-of-body experience. An identity problem. I was not in my body. I was in Jane's (another patient). No, she was in me. She was sitting in me. I don't know where I was. I couldn't locate myself. One thing for certain-I wasn't in my body' " (Gabbard \& Twemlow, 1984, p. 86). In this case, Miss G. had a particular problem differentiating herself from Jane. Jane, a self mutilator, would sometimes induce Miss G. also to mutilate herself. And, in fact, the therapist was asked never to bring up Jane again in case Miss G. felt she would have to duplicate her experiences, which had included an attempted enucleation of her eye.

The first main point of differentiation between the OBE and the schizophrenic body boundary disturbance is that of reality testing. Many of the signs of reality distortion are present in schizophrenic patients, including hallucinations, delusions, and self referential thinking. Reality testing is, however, intact in the out-of-body subject, who is free generally of other signs of major mental illness (Gabbard \& Twemlow, 1984; Jones, Gabbard \& Twemlow, 1984). The OBE is usually short-lived, in comparison with schizophrenic perceptual distortions, which are often chronic, and there is much more variation in these perceptual distortions in schizophrenic patients. These very varied possibilities should be contrasted with the highly specific and unvaried alteration of the mind/body relationship in the OBE subject. The location of the physical body is usually very clear to the OBE 
subject, often being seen below and slightly in front; whereas the schizophrenic often has great difficulty in identifying the location of the body, since the patient may often imagine that he or she has been engulfed and is now contained in the body of another individual. A major difference between the two includes the sense of identity, the out-of-body subject being stable and the schizophrenic being very fragile. The out-of-body subject often experiences the OBE as integrating and transforming in a positive rather than disorganizing sense; whereas the schizophrenic subject is likely to feel he or she is going crazy and to be in a state of great panic and psychotic anxiety.

\section{OBE and Organic Brain Syndrome}

OBEs have been associated in the literature with psychedelic drugs, certain drugs used to treat other illnesses, and with migraine headaches and temporal lobe epilepsy. N. Lukianowicz (1967) reported experiences in two patients treated wiht haloperidol and in one patient treated with alphamethydopa. As early as 1859, Paolo Mantegazza described OBEs during cocaine intoxication. In his own cocaine intoxication he felt he was floating throught the air out of his body amid colorful visions. Hallucinogens, such as LSD and mescaline, also produce bodily distortions and OBEs. The subjects in our study (Gabbard \& Twemlow, 1984) had a general low rate of drug usage: $75 \%$ of the population had never used marijuana, $89 \%$ had never used psychedelic drugs, and $51 \%$ reported never using any other category of nonprescription drugs. This particular population was quite old (average age of 48 years) and conservative. Other investigators have reported an association between marijuana use and OBEs, for example Charles Tart (1971). However, that association does not prove a causal link. In Tart's sample, about three quarters of the OBEs occurred in conjunction with marijuana or LSD, but the evidence that drug usage will make a person more open to the experience is lacking. Other cases of OBEs associated with temporal lobe epilepsy were described by Celia Green (1968) and Wilder Penfield and Herbert Jasper (1954), who reported that out-of-body impressions were evoked by electrical stimulation of the right temporal cortex. Migraine, and its link with the OBE, has been investigated by a variety of people, including Robert Crookall (1972) and Caro Lippman (1953), whose results, over a series of student samples, suggested that a history of migraine was more common in the out-of-body group than in the non-out-of-body group. Irwin's (1983) study, however, suggests that that association is spu- 
rious. From a clinical point of view, the important issue here is that a variety of organic brain states can produce out-of-body-like experiences and also classical OBEs. Thus, in each of these instances, the treatment of the underlying condition is required and the OBE itself is an incidental epiphenomenon of the fundamental clinical problem.

\section{The Impact of the OBE on the Individual}

By now it should be clear that the idea of "treatment" of the OBE is a misnomer. The OBE is a normal phenomenon of everyday psychology, requiring no treatment as such. I have taken great pains to distinguish the OBE from pathological states that require specific treatments in and of themselves, primarily to avoid two major pitfalls. One is the potential danger of certain treatments if used erroneously in normal individuals. These would include the undesirable side effects of antipsychotic drugs and the personally distressing and even disorganizing effect on the individual of the clinician treating this normal experience as abnormal. Subsequent to the publication of our book (Gabbard \& Twemlow, 1984), we have received numerous reports from individuals who are relieved that these experiences have been brought within the purview of normal everyday psychology. Case after case tells how these experiences, when reported to others and to physicians, were treated as signs of impending psychiatric illness. I have had numerous consultations with physicians who have considerable expertise in altered states of consciousness, who even now are not fully aware of the place of the OBE as a part of normal psychology.

The second feature of the management of the OBE has to do with its potentially transformative impact. The role of the modern clinician, in our view, is not only to treat existing disease, but to encourage the individual in healthy pursuits. Elsewhere (Twemlow \& Chamberlin, 1981) we have noted the increasingly important role of the clinician in involving him- or herself in activities that promote healthy living before disease afflicts the individual. This holistic approach can be applied equally to the management of the OBE. Merely to reassure the patient that he or she is not crazy would be, in some instances, to undermine a potentially very therapeutic and uplifting experience for the subject. In our original survey, $55 \%$ of the subjects experienced joy and $60 \%$ felt that their life was changed by the OBE, with $78 \%$ seeing a long-lasting benefit, with a change toward the belief in life after death in $66 \%$ of cases. Also, $43 \%$ of the subjects called the OBE "the greatest thing that ever happened to me" and $86 \%$ felt that they developed a 
greater awareness of reality, with a lasting benefit in $78 \%$ (Twemlow, Gabbard, \& Jones 1982). Although there was not a significant shift to any particular religious discipline, there was a significant shift toward the less traditional religious disciplines following the experience. Time after time, subjects described a striking shift in some of their existential concerns with an intensely focused introspective post-OBE phase. How am I doing? Who am I? Why am I here? Where am I going? and What is the meaning of my life? were significantly more frequently discussed by these subjects. That was, to me, even more astounding since the phenomenology of the typical OBE is quite mundane.

Although statistical data demonstrating the life-transformative effect of the OBE are lacking, in the case of the much more dramatic near-death experiences (NDEs), some research has reported an effect on personal values (Greyson, 1983). An out-of-body phase is often reported as a preliminary to the more dramatic NDE. Anecdotal accounts report life-transforming experiences, particularly the idea that an "off track" life gets "on track" after the experience. Bruce Greyson's study did not show that near-death subjects shared more spiritual or transformative values, although the control group sample, who had a high interest in NDEs, was quite biased against showing any differences between the groups. Greyson showed that the survivors of NDEs reported less interest in material and social success than control subjects. In another study by Greyson (1981), there was a negative attitude toward suicide after a NDE. Although these and other studies need to be broadened, there is at least the indication that some of these experiences may have clinically valuable psychotherapeutic effects. It is as if the experience is a form of rapid self-therapy. Thus, the clinician has an outstanding opportunity to help the patient grow and possibly prevent later illness by encouraging discussion and exploration of its impact. The informed clinician should encourage the patient to read literature and to continue an open discussion on the subject.

In previous work (Gabbard \& Twemlow, 1984) we have demonstrated how the ego psychological framework of Paul Federn (1952) is very useful conceptually for understanding these experiences. Federn demonstrated that the bodily and mental ego cathexis, although ordinarily fused and firmly integrated, can under certain conditions dissociate from one another. For example, he mentioned dissociation of the mental and bodily ego in the dream states, gradual loss of consciousness as in fainting, and various sleep transitions. Using this, we have elaborated a metapsychological understanding we call the ego uncoupling model. It is this healthy dissociation between mental and bodily ego that may encourage the sort of tranformative self reflection that is 
reported in subjects. In 91 subjects who were asked why they wanted to have an $\mathrm{OBE}, 25.3 \%$ were involved in existential explorations associated with major developmental stages in their lives, indicating a group who could well have been helped by an approach based on this ego uncoupling model.

\section{A Psychoeducational Approach to the OBE and Related Phenomena}

There is a large market in the lay press for techniques to induce the OBE. These techniques are being exploited by a variety of New Age entrepreneurs and also a number of serious research workers. Interest in these states, although in part perhaps motivated pathologically, may also reflect an interest people have in experiences that may help them deal with exigencies of everyday life, giving them a new perspective and reintroducing excitement and a sense of anticipation into their lives. While these phenomena do not necessarily come under the rubric "clinical," they are phenomena that the informed clinician is asked for advice about, especially as his or her role is expanding to include health-related as well as disease-related activities.

Over the past 20 years, I have evolved a series of clinical techniques to assist the individual in maximizing the understanding that he or she might achieve by these and other psychic experiences. I have called the approach psychoeducational. It is best conceptualized as a teacher/ pupil relationship rather than as a therapist/patient one, although there are qualities that frequently imbue it with features also similar to that of mentor/mentee. If the transformative experience is very intense or the follow-up to it causes radical change in the individual's lifestyle, there is then likely an attribution of some of this change to the teacher/mentor. The therapist must be very careful to recognize and address this idealizing relationship. Psychoanalytically informed clinicians will have no particular trouble in handling these transference phenomena; for the sake of those less familiar with psychoanalytic jargon, a word or two on transference seems in order. Many clinicians see transference as universal and as the basis of all human communication. In its simplest form, it involves attributing to others the traits of people from an earlier time in our life. These transferences from the past essentially bring ghosts of the past into the present and can often distort our way of relating to others. It is proposed, for example, that major transformative experiences may revive memories in the individual of the powerful thoughts and feelings that individual 
had towards his or her parents. Tranferences are unconscious phenomena and can catch the unaware therapist off guard. Some therapists treated thus begin to see themselves as god-like, to their own and the client's detriment. The New Age literature is replete with stories of the fallen guru and the abused disciples, leaving a quagmire of devastating human suffering and human feelings in its wake. With these ideas in mind, it is then possible to embark on a psychoeducational approach with appropriate awareness of the possibilities for transferential distortions of the relationship.

Once opened up to these realities by an $\mathrm{OBE}$, the individual then can begin the search with the assistance of a clinician. I have found it useful to expose the individual in an unbiased way to a variety of techniques that can help expand consciousness or insight.

These may be grouped as follows:

1. Meditative approaches: Formal training in meditation is available from reputable teachers. Especially widespread are transcendental meditation and others including the increasingly popular insight style of Vipassanna. Meditative states of consciousness are often very helpful in assisting the integration of the experience and in giving the individual an impetus to continue self-exploration. Those who find it difficult to sit may be eased into the meditative approaches through more active pursuits that encourage internal self-reflection, such as the martial arts and a variety of creative movement techniques, including dance. These techniques should not become ends in themselves, but merely ways in which the individual can integrate the experience.

2. Biofeedback therapies: For those more oriented to machinery and scientific measurement, biofeedback may well be the path of choice. Starting with simple techniques like fingertip temperature regulation and frontalis muscle tension reduction, the subject can then progress to more elaborate techniques like brainwave EEG training. Frequently, a relaxed body will stabilize more alterations in consciousness, which can allow the individual a deeper and more extensive exploration of himor herself.

3. Self-suggestion and other structured techniques: Techniques that rely on the suggestion of deep relaxation, including selfhypnotic techniques, are sometimes of value to individuals who require a more directed or structured technique less culturally different than the unusual conceptual frameworks be- 
hind the meditative disciplines. Thus, being given specific ways to relax by suggestion is of value. Besides self-hypnosis, techniques using binaural acoustical stimulation of the brain with accompanying suggestions about how to deploy internal attention are provided by a variety of techniques.

A further word is appropriate on the progression of the relationship in this process. Whereas it is of value during the selection of the technique suitable for the individual to have formal sessions at structured intervals, later the relationship should become more informal and on an as-needed basis. The mentor/mentee aspect of that relationship may continue over a number of years and a degree of informal supervision is often very helpful.

Needless to say, the use of these techniques is predicated on the personal experience and expertise the clinician has with these techniques before he or she attempts to use them with patients.

\section{References}

Blackmore, S. (1986). Out-of-body experiences in schizophrenia: A questionnaire survey. Journal of Nervous and Mental Disease, 174, 615-619.

Crookall, R. (1972). Casebook of Astral Projection. Secaucus, NJ: University Books.

Federn, P. (1952). Ego Psychology and the Psychoses. New York, NY: Basic Books.

Fisher, S., \& Seidner, R. (1963). Body experiences of schizophrenic, neurotic and normal women. Journal of Nervous and Mental Disease, 137, 252-257.

Gabbard, G.O., \& Twemlow, S.W. (1984). With the Eyes of the Mind. New York, NY: Praeger.

Gabbard, G.O., \& Twemlow, S.W. (1986). An overview of altered mind/body perception. Bulletin of the Menninger Clinic, 50, 351-366.

Green, C.E. (1968). Out-of-the-Body Experiences. London, England: Hamish Hamilton.

Greenhouse, H.B. (1975). The Astral Journey. Garden City, NY: Doubleday.

Greyson, B. (1981). Near-death experiences and attempted suicide. Suicide and LifeThreatening Behavior, 11, 10-16.

Greyson, B. (1983). Near-death experiences and personal values. American Journal of Psychiatry, 140, 618-620.

Haraldsson, E., Gudmundsdottir, A., Ragnarsson, A., Loftsson, J., \& Jonsson, S. (1977). National survey of psychical experiences and attitudes toward the paranormal in Iceland. In Morris, J.D., Roll, W.G., and Morris, R.L. (Eds.), Research in Parapsychology, 1976. Metuchen, NJ: Scarecrow Press.

Irwin, H.J. (1985). Flight of Mind. Metuchen, NJ: Scarecrow Press.

Jones, F.C., Gabbard, G.O., \& Twemlow, S.W. (1984). Psychological and demographic characteristics of persons reporting out-of-body experiences. Hillside Journal of Clinical Psychiatry, 6, 105-115.

Kennedy, R. (1976). Self-induced depersonalization syndrome. American Journal of Psychiatry, 133, 1326-1328.

Lippman, C.W. (1953). Hallucinations of physical duality in migraine. Journal of Nervous and Mental Disease, 117, 345-350. 
Lukianowicz, N. (1967). "Body image" disturbances in psychiatric patients. British Journal of Psychiatry, 113, 31-47.

Monroe, R.A. (1977). Journeys Out of the Body (2nd ed.). New York, NY: Anchor Books. Muldoon, S., \& Carrington, H. (1969). The Phenomenon of Astral Projection. New York, NY: Samuel Weiser. (Original work published 1951).

Noyes, R. Jr., Hoenk, P., Kuperman, S., \& Slymen, D. (1977). Depersonalization in accident victims and psychiatric patients. Journal of Nervous and Mental Disease, 164, 401-407.

Palmer, J. (1978). The out-of-body experience: A psychological theory. Parapsychology Review, 9(5), 19-25.

Penfield, W., \& Jasper, H. (1954). Epilepsy and the functional anatomy of the human brain. Boston, MA: Little, Brown.

Rawcliffe, D.H. (1959). Illusions and Delusions of the Supernatural and the Occult. New York, NY: Dover. (Original work published 1952).

Tart, C.T. (1971). On Being Stoned: A Psychological Study of Marijuana Intoxication. Palo Alto, CA: Science and Behavior Books.

Tobacyk, J.J., \& Mitchell, T.P. (1987). The out-of-body experience and personality adjustment. Journal of Nervous and Mental Disease, 175, 367-370.

Twemlow, S.W., Gabbard, G.O., \& Jones, F.C. (1982). The out-of-body experience: A phenomenological typology based on questionnaire responses. American Journal of Psychiatry, 139, 450-455.

Twemlow, S.W., \& Chamberlin, C.R. (1981). Holistic medicine. Journal of the Kansas Medical Society, 82, 447-450, 474. 